\title{
Network flow - Theory and applications with practical impact
}

\author{
Masao Iri \\ Department of Information and System Engineering \\ Faculty of Science and Engineering, Chuo University \\ 1-13-27 Kasuga, Bunkyo-ku, Tokyo 112, Japan. \\ Tel: +81-3-3817-1690. Fax: +81-3-3817-1681. \\ e-mail: iri@ise.chuo-u.ac.jp
}

Keywords

Network flow, history, applications, duality, future

\section{INTRODUCTION}

The research in this subject has now a long history and a large variety of related fields in science, engineering and operations research, and is still developing fast, especially in its algorithmic aspects, attracting many excellent senior as well as young researchers. And the space allotted to this paper is limited. So, in this paper I will not lay so much stress on particular frontiers of research in the subject as on the wider and historical viewpoint.

Network-flow theory is one of the best studied and developed fields of optimization, and has important relations to quite different fields of science and technology such as combinatorial mathematics, algebraic topology, electric circuit theory, nonlinear continuum theory including plasticity theory, geographic information systems, VLSI design, etc., etc., besides standard applications to transportation, scheduling, etc. in operations research.

An important point of view from which we should look at network flow theory is the "duality" viewpoint, to which due attention was paid at the earlier stages of development of the theory but which tends to be gradually ignored as time goes.

Since the papers and books published on network flow are too many to cite here, I do not intend to compile even an incomplete list of references but only to cite a few which I directly mention and which I think tend to become obscured during the long history in spite of their ever lasting importance. Happily enough, we saw a great book (Ahuja, Magnanti and Orlin, 1993) recently published on network flows containing most up-todate informations, as well as an extensive bibliography, on related mathematical theories, algorithms and applications. So, I will try to make the overlap of this survey paper of mine with this book as little as possible, i.e., to make the paper complementary to this book as much as possible. As may be natural, the standpoint of my survey is substantially 
influenced by the rather old book of mine (Iri, 1969), and details of a number of topics which are only briefly mentioned here will be found in this book.

\section{WHAT IS NETWORK FLOW?}

A flow network $N=(G ; \xi, \eta, \zeta, \cdots)$ is a graph $G=\left(V, E, \partial^{+}, \partial^{-}\right)$on which real quantities called flows $\xi$, tensions $\eta$ and potentials $\zeta$ are attached to its edges $E$ and vertices $V$ with the Kirchhoff laws satisfied, where some additional constraints are imposed on flows and tensions, and an appropriate objective (or objectives) is usually assumed. (See Figure 1.)

$$
\begin{array}{ll}
G=\left(V, E, \partial^{+}, \partial^{-}\right) & V: \text { vertex set, } E: \text { edge set } \\
& \partial^{+}: E \rightarrow V, \quad \partial^{-}: E \rightarrow V \quad \text { (incidence functions) } \\
N=(G ; \xi, \eta, \zeta, \cdots) & \xi: E \rightarrow \mathbf{R}, \quad \eta: E \rightarrow \mathbf{R}, \quad \zeta: V \rightarrow \mathbf{R}
\end{array}
$$

Kirchhoff's laws:

$\sum\left\{\xi(e) \mid e \in E, \partial^{+} e=v\right\}=\sum\left\{\xi(e) \mid e \in E, \partial^{-} e=v\right\} \quad$ for $\forall v \in V$

$\exists \zeta: \eta(e)=\zeta\left(\partial^{+} e\right)-\zeta\left(\partial^{-} e\right) \quad$ for $\forall e \in E$.

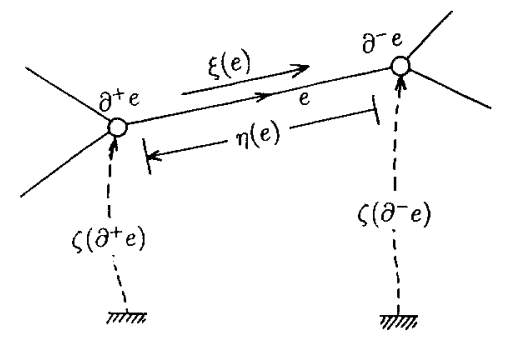

Figure 1 Basic elements of a network.

The most typical three network flow problems are

$1^{\circ}$ Maximum Flow Problem,

$2^{\circ}$ Shortest Path Problem,

$3^{\circ}$ Minimum-Cost Flow/Tension Problem,

each of which admits a natural interpretation in applications.

\section{DAWN OF THE THEORY}

Although there had been a number of earlier pioneering works, we owe the present-day formalism of network flow theory essentially to works by Minty, Fulkerson, Berge, et al. in the 1950-1960's, which are found in (Minty, 1960), (Berge et Ghouila-Houri, 1962), (Ford and Fulkerson, 1962), (Minty, 1966). Personally I should be glad if I were allowed to count the already-mentioned book of mine (Iri, 1969) among them. 


\section{EXPANDING HORIZONS OF APPLICATION}

Once the basic formalism of the theory was established, the horizons of application rapidly expanded to various fields of operations research such as assignment, transportation and scheduling, and even to branches of mathematics itself such as combinatorial mathematics. However, from the standpoint of more application-oriented people including myself, this type of statement may seem to be inappropriate. It might better be stated that accumulation of a pile of individual practically solved examples and unsolved needs motivated people to establish a novel theory. Among those examples we may count the now "prehistoric" works such as (Monge, 1781) and (Kantorovitch, 1942).

\section{RELATED FIELDS}

There are a number of classical as well as modern fields of physical sciences and engineering where the same or similar logical structure is found as network-flow theory. In particular, the theory of nonlinear monotone resistive electric networks is exactly the same as (single-commodity) network-flow theory, although, regrettably, there is sometimes allergy to "electricity" among network-flow people - at least around me in Japan.

It is interesting to note that, in the earlier age of development of network-flow theory, "electrical" viewpoint was emphasized by a few people almost simultaneously, e.g. , in (Dennis, 1959), (Iri, 1959), (Minty, 1960).

In an electric network whose topology is determined by a graph $G=\left(V, E, \partial^{+}, \partial^{-}\right)$,

electric current $\xi(e)$ in edge $e \in E$ is interpreted as the flow in it, electric voltage $\eta(e)$ across edge $e \in E$ as the tension across it,

and

electric potential $\zeta(v)$ at vertex $v \in V$ as the potential there.

For each edge $e \in E$ the current/flow and the voltage/tension are related to each other in terms of a monotone nondecreasing function, of which noteworthy special cases are shown in Figure 2.

An electric network to represent a (two-terminal) maximum flow problem can be composed of a single edge of type (ii) (whose two end vertices represent the two terminals of the network) and all the other edges of types (i) and (iii). An electrical network to represent a (two-terminal) shortest path problem can be composed of a single edge of type (i) and all the other edges of types (ii) and (iii). An electric network to represent a minimum-cost flow problem (including transportation, assignment, etc.) as well as a minimum-cost tension problem (including PERT/CPM-type scheduling problems, ete.) can be composed of edges of types (i), (ii) and (iii).

All these problems are "linear" network flow problems, but the electrical networks for them contain "highly nonlinear" elements, namely, diodes. If edges of type (iv) (which are most popular "linear" elements in electric networks) are contained in the network, then we shall have a "quadratic-cost" network flow problem. 
(i) currrent source for which $\xi(e)=$ given constant irrespective of $\eta(e)$ :

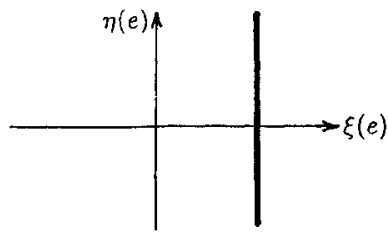

(ii) voltage source for which $\eta(e)=$ given constant irrespective of $\xi(e)$ :

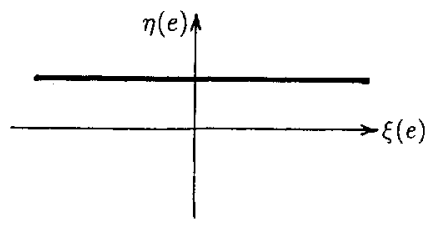

(iii) diode (inequality) for which $\xi(e) \geq 0, \eta(e) \geq 0, \xi(e) \cdot \eta(e)=0$ :

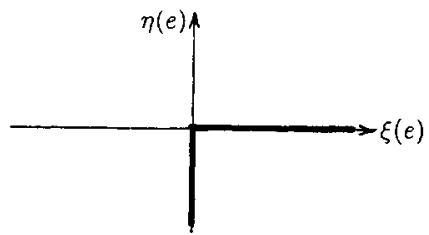

(iv) linear resistor for which $\eta(e)=r(e) \xi(e)$

where $r(e)$ is the resistance (a positive constant):

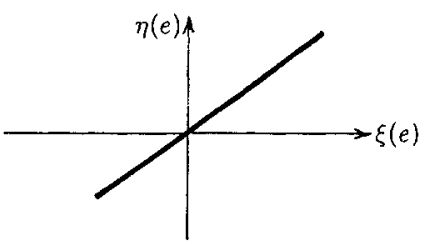

Figure 2 Typical flow-tension (or current-voltage) relations.

\section{ALGORITHMS DEVELOPED}

Around 1970 there was a discontinuous progress in graph and network-flow algorithms owing to Hopcroft and Tarjan's epoch-making works. Since then, a tremendously large number of works have been done to make the related algorithms faster and faster, and now we have a big family of network algorithms some of which make use of highly sophisticated data-structures sometimes adversely called "acrobatic".

Since linear network-flow problems can be solved much faster by means of proper al- 
gorithms than the corresponding linear-programming problems are solved by means of the simplex or interior-point algorithms, and that the tractable problem size is far larger, the reducibility of a linear-programming problem to a network-flow problem naturally attracts the researchers' interests. Here again, it is interesting to note that this reducibility problem is equivalent to the so-called "graph-realization problem" which has been studied for the purpose of application to the topological synthesis of electrical networks.

The history of research on this topic is long, starting from those in early days, such as (Iri, 1962), (Iri, 1966), (Iri, 1968), through a number of researches, e.g. , (Bixby and Cunningham, 1980), until the latest, e.g. , (Gautier and Granot, 1994). Probably, (Fujishige, 1980) gives a best result for the graph realization algorithm.

\section{SINGLE-COMMODITY VS. MULTICOMMODITY}

There are more practical problems which are modelled as a multicommodity flow problem than those modelled as a single-commodity flow problem, but from the point of view of solution algorithm, the former shares the advantage of the latter only partially. This is mainly because the nice property of "total unimodularity" possessed by the singlecommodity flow is not valid for the multicommodity any longer.

Although there have been a lot of progress in algorithmic and technical aspects of solutions of multicommodity flow problems, the old survey (Jewell, 1966) still remains to be an excellent general introduction to multicommodity flow problems.

Traditional empirical "incremental assignment" algorithms developed and used by rather practical traffic assignment people seemingly motivated a number of decomposition principles and column-generation techniques, and the incremental-type algorithms have become theoretically better grounded. Efficient solution algorithms have been found for special classes of problems including various VLSI design problems.

There are the dual counterparts (see $\S 8$ ) of multicommodity flow problems which may be called "multi-commodity tension" problems and which represent some characteristics of multi-job scheduling problems, although they are less popular.

\section{DUALITY}

The duality is one of the most conspicuous features of network-flow theory. One can better understand the theory with the concept of duality and apply the theory to wider practical fields than without it. It seems that more stress was laid on the duality in the earlier stages of development of the theory than nowadays. (Berge et Ghouila-Houri, 1962) was dualityconscious, whereas (Ford and Fulkerson, 1962) was less conscious. Viewed from matroid theory (cf. (Minty, 1966)) the duality is a very natural structure of network-flow theory, i.e., that "part" of network flow theory which can be stated in terms of concepts related to edges only without reference to vertices possesses a "dual" logical structure. This duality of the theory comes from the duality of graph theory, and every proposition for flows has its dual counterpart for tensions, and vice versa.

Sometimes the concept of the "duality of the theory" (i.e., the dualistic structure of the theory itself, which is a "meta-theoretical" concept as it were) is confused with the "duality theorem" on optimization problems or their "dual problems", i.e., with the 
"mathematical-programming duality". But the two must be clearly distinguished from each other although they may coincidentally lead us to one and the same pair of propositions for some problems. Thus, in some cases, for a pair of dual problems or duality theorems in the sense of mathematical programming, we may have the meta-theoretically dual pair, i.e., we may have a quadruple of problems or theorems. Figure 3 gives an example.

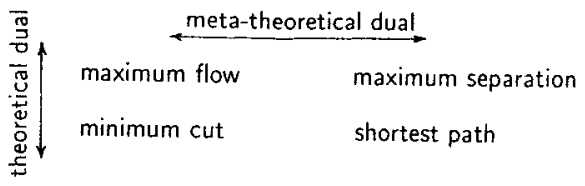

Figure 3 Two kinds of duality.

graph/network

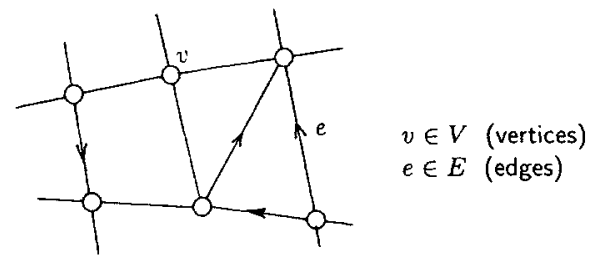

$\operatorname{map} /$ GIS

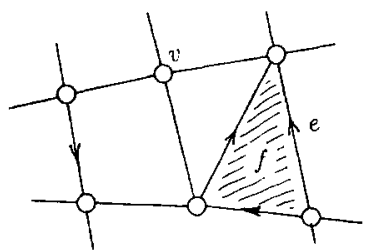

$v \in V$ (vertices)

$e \in E$ (edges)

$f \in F$ (faces)

3-dim. GIS

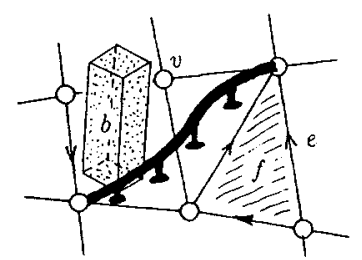

$v \in V$ (vertices)

$e \in E$ (edges)

$f \in F$ (faces)

$b \in B$ (boxes)

4-dim. GIS

"history of temporal changes"

Figure 4 From network to 4-dimensional GIS. 
In addition, it is worthwhile to note that traditionally well-known "triangular inequalities" for distances $d_{\alpha \beta}$ 's (between pairs of vertices in a network) and for terminal-pair capacities $c_{\alpha \beta}$ 's (in a capacitated network) $\left(v_{\alpha}, v_{\beta}, v_{\gamma} \in V\right)$ :

$d_{\alpha \gamma} \leq d_{\alpha \beta}+d_{\beta \gamma}, \quad c_{\alpha \gamma} \geq \min \left(c_{\alpha \beta}, c_{\beta \gamma}\right)$

are not the meta-theoretical dual of each other, so that we do have two dual counterparts of theirs. To find them will be an enjoyable exercise. See, for details, (Iri, 1981).

\section{EXTENSIONS}

Techniques and, what is more important, spirits, fostered for network flows are extended in various directions.

\subsection{Dimension}

Topologically, a graph may be regarded as a one-dimensional topological complex. Extension in dimension will lead us to 2-dimensional complexes, of which the simplest but practically most useful examples are map data systems or GIS (geographic information systems, now becoming a big industry). The latter may be extended to 3-dimensional GIS to deal with underground and sky information, and further to 4-dimensional GIS to deal also with time sequences or temporal changes. (See Figure 4.)

\subsection{Underlying algebraic structure}

With respect to the shortest-path problem which makes a simplest class of network-flow problems, the so-called "min-addition algebra" plays an interesting role. It is an algebraic structure on the set of real numbers and $\infty$ in which ordinary "addition" and "multiplication" are replaced, respectively, by "min" and "addition". Earlier references may be (Carré, 1971a), (Carré, 1971b). Extensions and applications of this kind of algebraic structure have been intensively studied by Burkard's school in Graz. (See also §9.3.)

Mechanical structures such as trusses and frames may be regarded as an extension in this direction where vector-tensor flows and tensions are attached to the underlying graph, and the graph is not merely a "topological" existence but "geometric" in the sense that the continuity of forces and moments as well as the compatibility of deformations (corresponding to Kirchhoff's laws for ordinary flows and tensions) is expressed in terms not only of the topology of the structure but also of its geometry, i.e., lengths and angles.

\subsection{Leaky Kirchhoff}

Network flows with gains and losses come out by making Kirchhoff's laws "leaky" (see Figure 5). An earlier but rather complete presentation is found in (Jewell, 1962).

It is interesting to see the "min-addition algebra" naturally extended to this kind of problems to produce a new class of algebras which, probably, has been given no proper name yet (Iri, Amari and Takata, 1961), (Iri, Amari and Takata, 1968).

This extension was obviously motivated by application to machine loading problems, which we may find in the monumental book on operations research, i.e., (Churchman, 
Ackoff and Arnoff, 1957), and rather extensive studies by (then) Roumanian group ( $\mathrm{K}$. Eisemann, J. R. Lourie, E. Balas and P. L. Ivanescu) are found in three papers (pp. 154202) of Vol. 11, No. 1 (1964) of Management Science.

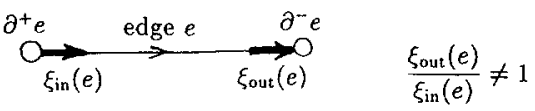

Figure 5 Edge with gain/loss.

\subsection{Extra constraints}

A small number of extra constraints imposed on an ordinary network flow problem can be treated without seriously deteriorating the efficiency of the solution algorithms. Thus flows with bundle constraints as well as tensions with bundle constraints were studied in earlier days. The idea of flow bundle is found in (Berge et Ghouila-Houri, 1962) and that of tension bundle (although not so named) in (Jewell, 1965). This type of problems seems still attracting researchers' interest (see, e.g. , (Gautier and Granot, 1994)). Further extension is to consider "submodular" constraints on flows in edges (Fujishige, 1991).

However, if we admit to introduce a large number of bundle constraints and to make Kirchhoff's laws leaky, we shall be led to fairly general optimization problems (containing linear programming problems), Rockafellar's "monotropic optimization" being essentially of this type (Rockafellar, 1984).

\subsection{Multiple objectives}

Compromise between two (or more) conflicting objectives is an interesting research subject which is important in application. For example, the problem of choice between "time" and "cost" when we use a high-way network is of this kind. We can trace the origin of the idea back to (Lawler, 1967), at least, and the extension in this direction will add a flavour of "fractional programming" to network flows. See, e.g. , (Megiddo, 1979).

\subsection{Discretum to continuum}

One of the earliest problems in the history of optimization was the continuous transportation problem (Monge, 1781), (Kantorovitch, 1942). It was a problem on flows in a field, not in a network. But recent trend of research consists in "generalizing" results in network flow theory to flows (and tensions) in continua. Also, there are interesting and challenging problems of approximating a continuum (i.e., field) by a discretum (i.e., network) and vice versa. (See (Iri, 1980), (Taguchi and Iri, 1982).)

It seems that there are several different approaches to model, or define, a problem of flows in continua, e.g., (Hu, 1969), (Strang, 1983), (Blum, 1990), (Nozawa, 1994). 


\section{MONOTONICITY, NON-AMPLIFICATION, SENSITIVITY AND RECIPROCITY}

These concepts are familiar in electric network theory but not so much in network flow theory. However, in principle, the concepts themselves as well as the related results in the former theory can be transferred to the latter. They may be summarized as follows.

$1^{\circ}$ Monotonicity:-- If the flow in and the tension across every edge of a network are in monotone nondecreasing relation to each other, then, between any two vertices in the network, the flow and the tension are also in a monotone nondecreasing relation, i.e., the two-terminal characteristic is monotone.

$2^{\circ}$ Non-amplification:-- If a current source of value $\varepsilon$ is attached in parallel to an edge ( $\epsilon . g .$, the capacity of the edge is perturbed by $\varepsilon$ ), then the corresponding perturbation of the flow in any edge does not exceed $\varepsilon$ in absolute value (e.g., the value of the maximum flow does not change by more than $\varepsilon$ ). Dually, if a voltage source of value $\varepsilon$ is inserted in series to an edge (e.g., the length of the edge is perturbed by $\varepsilon$ ), then the corresponding perturbation of the tension across any edge does not exceed $\varepsilon(e . g$. , the shortest distance does not change by more than $\varepsilon$ ).

$3^{\circ}$ Sensitivity:- The sensitivity is the variation of flows and tensions against some perturbation in the flow-tension relation of an edge. The "out-of-kilter" method (called so in (Ford and Fulkerson, 1962) but the idea had been presented in (Minty, 1960) in a more general form) affords a method of calculating the variation concretely.

$4^{\circ}$ Reciprocity:- We assume that the pair of flow and tension of every edge of the network is at a point where the relation between them has a well-defined tangent (i.e., the relation is "differentiable" at that point). Let, furthermore, the variation in the tension across an edge $e_{j}$ when a current source of value $\Delta \xi\left(e_{i}\right)$ is attached in parallel to another edge $e_{i}$ be denoted by $\Delta \eta\left(e_{j}\right)$. Then we have

$$
\frac{\Delta \eta\left(e_{2}\right)}{\Delta \xi\left(e_{1}\right)}=\frac{\Delta \eta\left(e_{1}\right)}{\Delta \xi\left(e_{2}\right)}
$$

The dual statement is valid, too.

The common-sense "monotonicity" conjecture that any additional investment on a system will result in some improvement on the system function/performance sometimes fail$\mathrm{s}$, in particular for multicommodity flow networks. The famous Braess paradox (Braess, 1968) says that there is the case where construction of a new road will result in increase of each user's travel time, thus deteriorating the system performance in any sense. A few other paradoxes of similar kind are known in traffic assignment theory. Quite recently, a popular-science article (Arnott and Small, 1994) was published, which indicates that the problem is still of hot interest in the field of urban design.

The basic principles underlying traffic assignment are so-called "Wardrop's laws" (Wardrop, 1952), where two different concepts of optimality, i.e., "system optimization" and "user optimization", are enunciated and their mutual relations discussed. 


\section{RESTRUCTURING THE THEORY NEEDED?}

In ordinary network-flow theory, cancellation of flows in an edge is allowed - or even necessary in many algorithms of augmenting-path type - when two or more flows are superposed (or added), which is a barrier hard for the beginner to jump over. By making use of it, we can get an optimum plan. And we anticipate users of a system to obey the plan afforded by the planner. However, in the real world of the new century, which will certainly be a century of multiple values, we cannot expect users of a system to be obedient to the planner, i.e., we must be prepared to assume that users may use the system in whatever way physically possible. In such circumstances, cancellation of flows will not be a realistic operation. So, there arises an idea of restructuring, from scratch, network flow theory without allowing cancellation of flows. Then what will happen as the result of such restructuring? A lot remains to be investigated, but there is an essay in that direction (Iri 1994). Among a number of examples there, I will take up one.

In the two-terminal capacitated network of Figure 6 (left), where all the edges are one-way and the numbers beside edges indicate the capacities of edges, the value of a maximum flow in the ordinary sense is obviously equal to 2 . Let us then consider the unit flow along the path shown in Figure 6 (right). Since the flow saturates all the three edges on the path which together make a cut between the entrance vertex and the exit vertex, we cannot augment the flow any more without cancelling the flow in the vertical edge. Thus, the flow of Figure 6 (right) is a "maximal" (or "blocking", as is sometimes called) flow from the entrance to the exit - of the minimum value in this case.
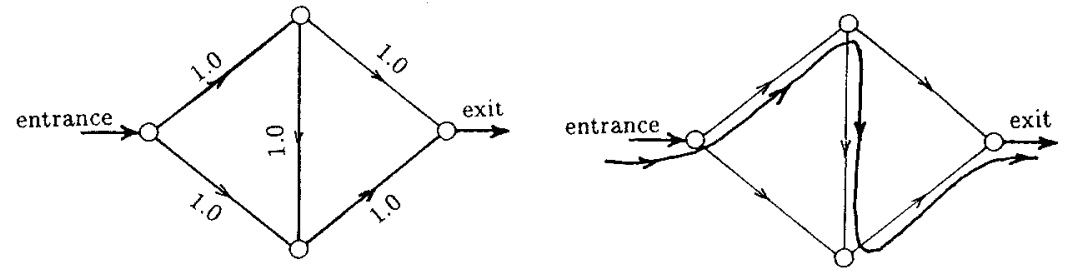

Figure 6 A capacitated network (left) and a unit flow along a path (right).

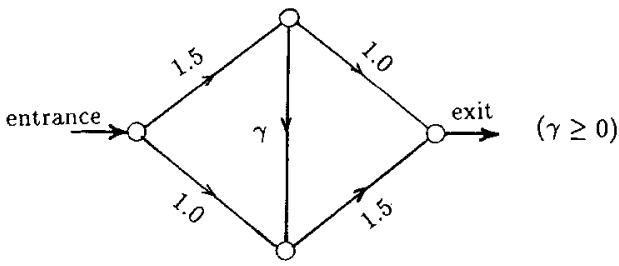

Figure 7 Another capacitated network with a parametric capacity $\gamma$.

It may be interesting to note that the dependence of the minimum value of maximal two-terminal flows on edge capacities is far from being monotone. For example, look at the network of Figure 7, where the numbers indicate edge capacities as before and $\gamma$ is 
the parametric capacity of the vertical edge. Then the dependence of the minimum value of maximal flows on $\gamma$ will be seen to be as shown in Figure 8.

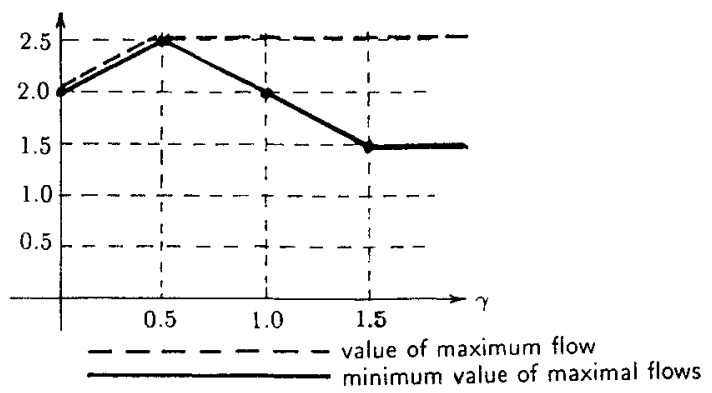

Figure 8 Minimum value of maximal flows against $\gamma$.

\section{REFERENCES}

Ahuja, R.K., Magnanti, T.L. and Orlin, J.B. (1993) Network Flows -- Theory, Algorithms, and Applications. Prentice Hall, Englewood Cliffs, New Jersey.

Arnott, R. and Small, K. (1994) The economics of traffic congestion. American Scientists, 82, 446-55.

Berge, C. et Ghouila-Houri, A. (1962) Programmes, Jeux et Réseaux de Transport. Dunod, Paris.

Bixby, R.E. and Cunningham, W.H. (1980) Converting linear programs to network programs. Mathematics of Operations Research, 5, 321-57.

Blum, W. (1990) A measure-theoretical max-flow-min-cut problem. Mathematische Zeitschrift, 205, 451-70.

Braess, D. (1968) Über ein Paradoxon aus der Verkehrsplanung. Unternehmensforschung, 12, 258-684.

Carré, B.A. (1971a) An algebra for network routing problems. Journal of the Institute of Mathematics and Its Applications, 7, 273-94.

Carré, B.A. (1971b) An elimination method for minimal cost network flow problem, in Large Sparse Sets of Linear Equations (ed. J.K. Reid), Academic Press.

Churchman, C.W., Ackoff, R.L. and Arnoff, E.L. (1957) Introduction to Operations Research. John Wiley \& Sons, New York, 377-9.

Dennis, J.B. (1959) Mathematical Programming and Electrical Networks. MIT Technology Press, Cambridge, Massachusetts, and John Wiley \& Sons, New York.

Ford,L.R., Jr., and Fulkerson, D.R. (1962) Flows in Networks. Princeton University Press, Princeton, New Jersey.

Fujishige, S. (1980) An efficient PQ-graph algorithm for solving the graph-realization problem. Journal of Computers and System Sciences, 21, 63-86.

Fujishige, S. (1991) Submodular Functions and Optimization. Annals of Discrete Mathematics 47, North-Holland, Amsterdam-New York-Oxford-Tokyo.

Gautier, A. and Granot, F. (1994) On the equivalence of constrained and unconstrained 
flows. Discrete Applied Mathematics, 55, 113-32.

Hu, T.C. (1969) Integer Programming and Network Flows. Addison-Wesley, Massachusetts.

Iri, M. (1959) Algebraic and topological theory of the problems of transportation networks with the help of electric circuit models. RAAG Research Notes, Third Series, No. 13.

Iri, M., Amari, S. and Takata, M. (1961) Linear programming with weak graphical representation. RAAG Research Notes, Third Series, No. 47.

Iri, M. (1962) A necessary and sufficient condition for a matrix to be a loop or cut-set matrix of a graph and a practical method for the topological synthesis of networks. RAAG Research Notes, Third Series, No. 50.

Iri, M. (1966) A criterion for the reducibility of a linear programming problem to a linear network-flow problem. RAAG Research Notes, Third Series, No. 98.

Iri, M. (1968) On the synthesis of loop and cutset matrices and the related problems. RAAG Memoirs, 4, A-XIII, 4-38.

Iri, M., Amari, S. and Takata, M. (1968) Algebraical and topological theory and methods of linear programming with weak graphical representation. $R A A G$ Memoirs, 4, MiscVII, 539-620.

Iri, M. (1969) Network Flow, Transportation and Scheduling - Theory and Algorithms. Academic Press, New York and London.

Iri, M. (1980) Theory of flows in continua as approximation to flows in networks, in Survey of Mathematical Programming (ed. A. Prékopa), (Proceedings of the IXth International Symposium on Mathematical Programming, Budapest, 1976), North-Holland, 2.

Iri, M. (1981) "Dualities" in graph theory and in the related fields viewed from the metatheoretical standpoint, in Graph Theory and Algorithms, (eds. N. Saito and T. Nishizeki), Lecture Notes in Computer Science 108, Springer-Verlag, Berlin-HeidelbergNew York.

Iri, M. (1994) An Essay in the Theory of Uncontrollable Flows and Congestion. TRISE (Technical Report on Information and System Engineering) 94-03, Faculty of Science and Engineering, Chuo University. (Also presented at the XVth International Symposium on Mathematical Programming, August 1994, University of Michigan, Ann Arbor, Michigan.)

Jewell, W.S. (1962) Optimal flows with gains. Operations Research, 10, 476-99.

Jewell, W.S. (1965) Divisible activities in critical path analysis. Operations Research, 13, $747-60$.

Jewell, W.S. (1966) A Primal-Dual Multi-commodity Flow Algorithm. Report ORC 66-24, OR Center, University of California, Berkeley, California.

Kantorovitch, L. (1942) On the translocation of masses. Comptes Rendus (Doklady) de l'Academie des Sciences de l'URSS, XXXVII, 199-201 (Mathematics).

Lawler, E.L. (1967) Optimal cycles in doubly weighted linear graphs, in Theory of Graphs (ed. P. Rosenstiehl), Dunod, Paris.

Megiddo, N. (1979) Combinatorial optimization with rational objective functions. Mathematics of Operations Research, 4, 414-24.

Minty, G.J. (1960) Monotone networks. Proceedings of the Royal Society, A251, 194-212.

Minty, G.J. (1966) On the axiomatic foundation on the theories of directed linear graphs, electrical networks and network-programming. Journal of Mathematics and Mechanics, 15, 485-520.

Monge, G. (1781) Déblai et remblai. Mémoires de l'Academie des Sciences. 
Nozawa, R. (1994) Examples of max-flow and min-cut problems with duality gaps in continuous networks. Mathematical Programming, 63, 213-34.

Rockafellar, R.T. (1984) Network Flows and Monotropic Optimization. John Wiley \& Sons, New York.

Strang, G. (1983) Maximal flow through a domain. Mathematical Programming, 26, 12343.

Taguchi, A. and Iri, M. (1982) Continuum approximation to dense networks and its application to the analysis of urban road networks. Mathematical Programming Study, 20, 178-217.

Wardrop, J.G. (1952) Some theoretical aspects of road traffic research. Proceedings of the Institute of Civil Engineers, 1, 325-78. 